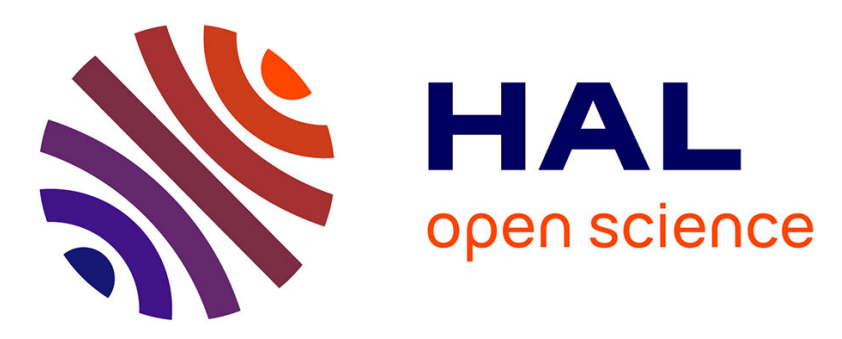

\title{
Etude de la dispersion de faisans d'élevage marqués dans un secteur du Gâtinais
}

\author{
Ctgref Centre Technique Du Génie Rural Des Eaux Et Forêts
}

\section{To cite this version:}

Ctgref Centre Technique Du Génie Rural Des Eaux Et Forêts. Etude de la dispersion de faisans d'élevage marqués dans un secteur du Gâtinais. Revue forestière française, 1972, 24 (5), pp.346-353. $10.4267 / 2042 / 20631$. hal-03395548

\section{HAL Id: hal-03395548 \\ https://hal.science/hal-03395548}

Submitted on 22 Oct 2021

HAL is a multi-disciplinary open access archive for the deposit and dissemination of scientific research documents, whether they are published or not. The documents may come from teaching and research institutions in France or abroad, or from public or private research centers.
L'archive ouverte pluridisciplinaire HAL, est destinée au dépôt et à la diffusion de documents scientifiques de niveau recherche, publiés ou non, émanant des établissements d'enseignement et de recherche français ou étrangers, des laboratoires publics ou privés. 


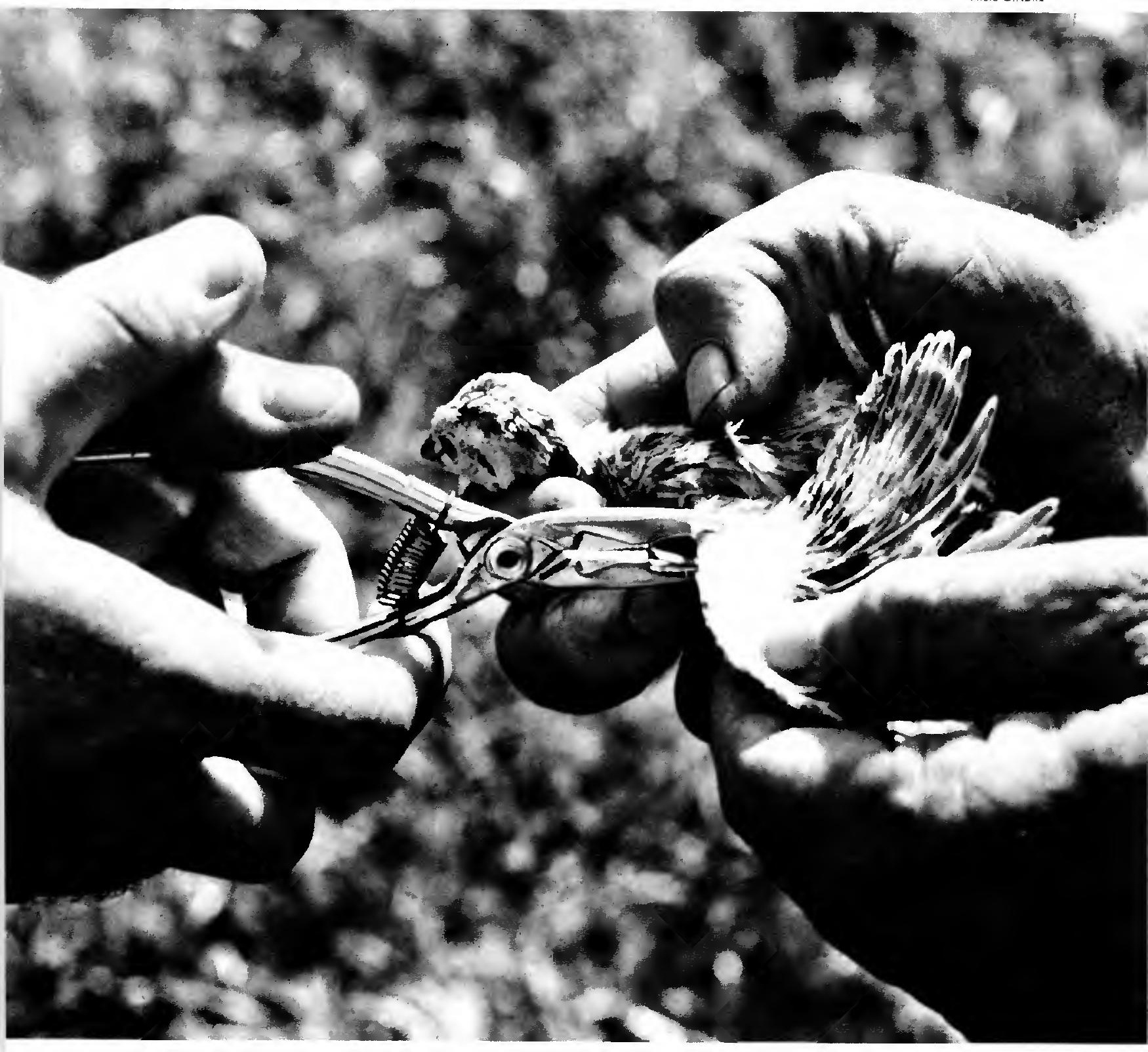




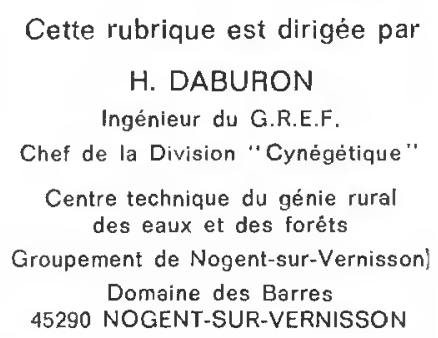

Cette rubrique est dirigée par

H. DABURON

Ingénieur du G.R.E.F.

Chef de la Division "Cynégètique"

Centre technique du génie rural des eaux et des foréts

Groupement de Nogent-sur-Vernisson)

Domaine des Barres

45290 NOGENT-SUR-VERNISSON

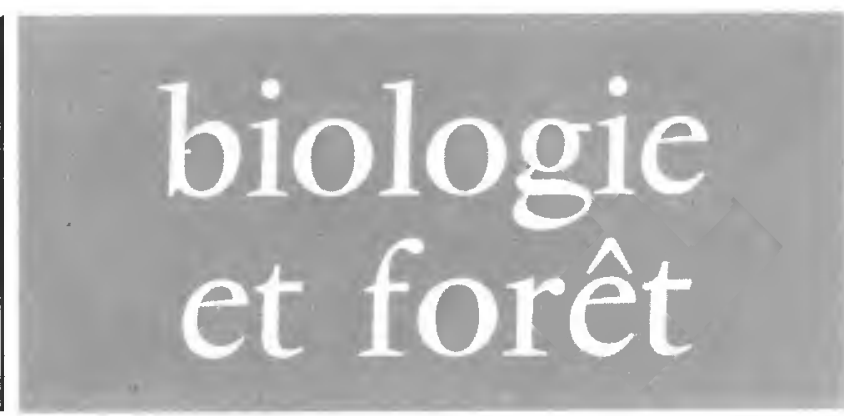

\section{ÉTUDE DE LA DISPERSION DE FAISANS D'ÉLEVAGE MARQUÉS DANS UN SECTEUR DU GÂTINAIS}

Class. Oxford 156.1/.2: 148.2 Phasianus colchicus L.

Résumé : Les premiers résultats d'une expérience en cours dans un secteur du Gâtinais montrent les possibilités pratiques d'études par marquage du devenir des faisans communs (Phasianus colchicus L.) d'élevage après leur lâcher sur un territoire de chasse. Le taux de reprise lors de la dernière saison de chasse, la dispersion après le lâcher et le sex ratio des oiseaux figurant au tableau de chasse sont comparés à des données analogues obtenues en Grande Bretagne.

\section{BUT DE L'EXPÉRIMENTATION}

Etudier par la technique du marquage le succès des lâchers de faisans d'élevage (Phasianus colchicus L.) et la dynamique des populations constituées par des oiseaux provenant en partie d'élevage et en partie de reproduction naturelle, en vue d'une meilleure gestion des territoires de chasse.

\section{DESCRIPTION DES EXPÉRIENCES}

\section{Territoire d'expérimentation}

Le secteur d'expérimentation est constitué par un territoire central et un territoire périphérique (plan). Le territoire central de 1.200 ha comprend les domaines de chasse des Barres (300 ha) et du Buisson (900 ha). Le territoire périphérique s'étend sur 2.500 ha environ.

\section{Marquage}

En 1971 tous les oiseaux d'élevage lâchés dans le territoire central étaient marqués à l'aile au moyen d'agrafes en acier inoxydable (tableau, p. 350). 


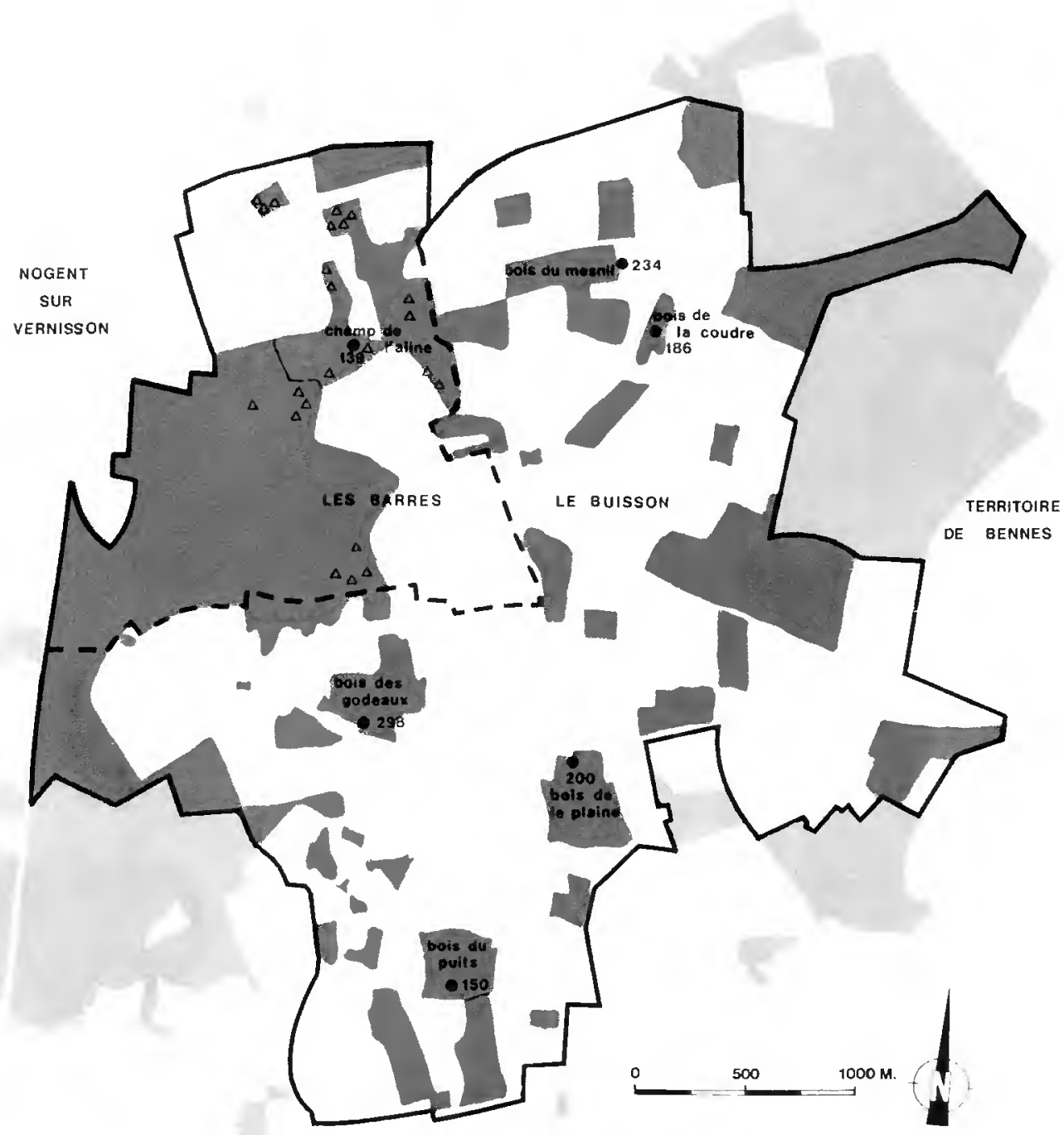

LEGENDE

- 234 POINT de lacher et nombre de faisandeaux

$\triangle \quad$ PIEges pour capturer les faisans

- LIMITE DU TERRITOIRE CENTRAL

- LIMITE BARRES-BUISSON

BOIS A L'INTERIEUR DU TERRITOIRE CENTRAL

BOIS A L'EXTERIEUR DU TERRITOIRE CENTRAL

\section{Elevage et lâchers}

6 groupes de marquage:

Groupes de 1 à 5 : marquage de 1068 poussins de 8 à 10 jours, puis mise au bois avec poules meneuses à l'âge de 3 semaines, entre le 15 juin et le 16 juillet.

Groupe 6 : marquage de 139 faisandeaux de 6 semaines éduqués en éleveuse artificielle, au moment de leur mise en volière d'acclimatation, une semaine avant le lâcher.

Les points de lâcher ne correspondaient chacun à un seul groupe de marquage que pour les groupes $1,2,3$ et 6 , les groupes 4 et 5 étant répartis entre 4 points de lâcher. 


\section{Contrôles}

Au cours de l'automne 1971 et de l'hiver 1971-1972 : contrôle total du territoire central, et partiel du territoire périphérique.

Territoire central : battue)

LE BUISSON : examen des tableaux de chasse à la fin de chaque traque (chasse en

- LES BARRES : capture au piège.

Territoire périphérique :

- BENNES, 500 ha à l'est du BUISSON : examen des tableaux de chasse

- 2.000 ha : contrôle partiel grâce au concours d'une trentaine de correspondants (propriétaires, gardes-chasse, chasseurs informés et visités régulièrement).

Lors de l'examen des tableaux de chasse et des captures au piège, le nombre et le sexe des faisans marqués et non marqués étaient enregistrés. Dans le territoire périphérique, le sexe de certains faisans marqués et tués n'a pu être enregistré.

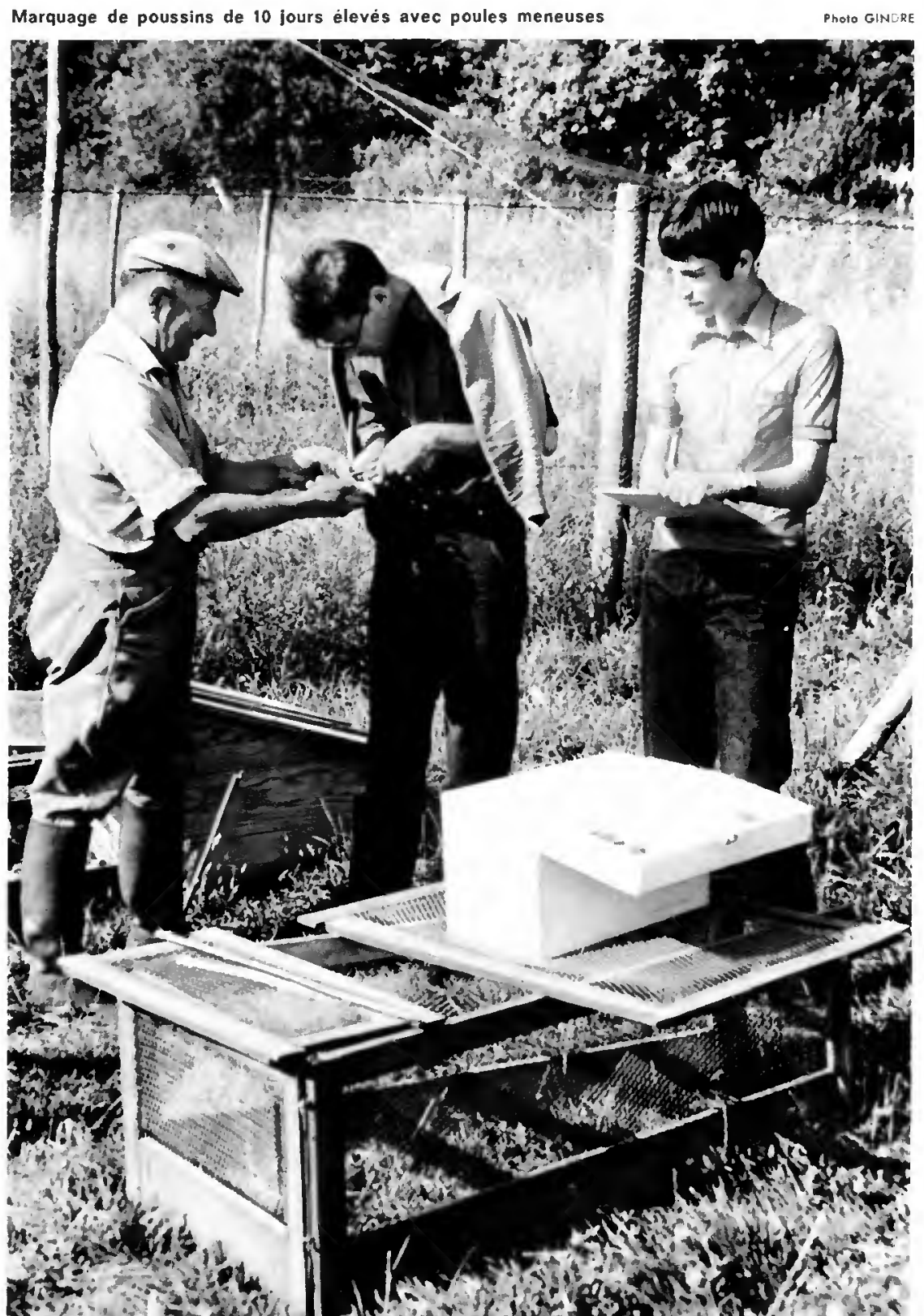




\begin{tabular}{|c|c|c|c|c|c|c|c|c|c|c|}
\hline \multirow{10}{*}{$\begin{array}{l}* \\
* \\
w \\
\frac{\sigma}{\sigma} \\
\frac{a}{a} \\
\underset{\sim}{\alpha}\end{array}$} & \multicolumn{2}{|c|}{ 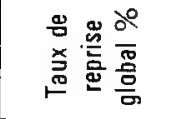 } & $\stackrel{\text { 尔 }}{8}$ & 里 & 吕 & $\begin{array}{l}0 \\
\text { g } \\
q\end{array}$ & $\overline{8}$ & $\bar{m}$ & $\underset{\sim}{\sim}$ & \multirow{13}{*}{ 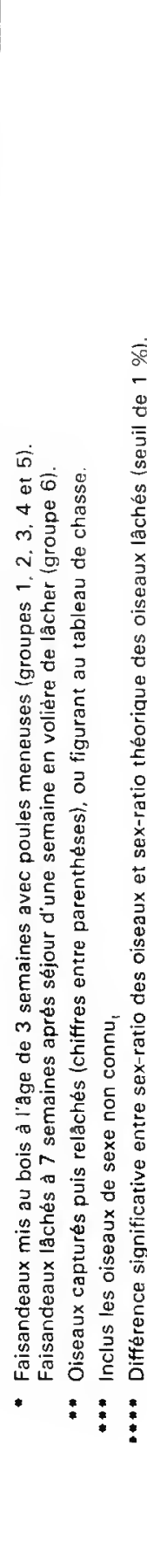 } \\
\hline & \multirow{4}{*}{ 这 } & 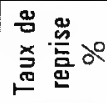 & $\tilde{0}$ & $\bar{\sim}$ & 0 & $\stackrel{a}{\sim}$ & $\stackrel{\sigma}{m}$ & $\stackrel{\square}{\sim}$ & $\stackrel{\infty}{+}$ & \\
\hline & & 垔: & $\stackrel{\llcorner}{\sim}$ & $N$ & 0 & $r$ & $m$ & $=$ & $\stackrel{\infty}{\infty}$ & \\
\hline & & 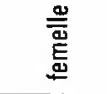 & 으 & - & & & תL & ص & $\bar{N}$ & \\
\hline & & $\frac{0}{\stackrel{0}{0.0}}$ & $\sigma$ & & & - & $m$ & مس & $\stackrel{\infty}{-}$ & \\
\hline & \multirow{5}{*}{ 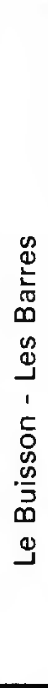 } & 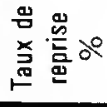 & $\stackrel{\infty}{\ddot{m}}$ & $\stackrel{\infty}{\stackrel{m}{m}}$ & 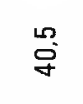 & $\underset{\infty}{+}$ & $\stackrel{N}{n}$ & 沗 & 吕 & \\
\hline & & 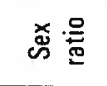 & 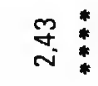 & $\stackrel{\text { 莒 }}{-}$ & $\cong$ & 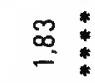 & 용 : & $\frac{\sigma}{\sigma}$ & 邑 & \\
\hline & & 预: & $\stackrel{\infty}{\frac{n}{2}}$ & $\stackrel{\stackrel{\infty}{\sim}}{\stackrel{\Xi}{n}+}$ & $\stackrel{\infty}{\stackrel{2}{\Sigma}}$ & $\begin{array}{l}\stackrel{\infty}{\Xi} \\
\stackrel{5}{\frac{0}{0}} \\
\frac{0}{=}\end{array}$ & $\frac{\stackrel{2}{\simeq}}{\frac{\pi}{ \pm}}$ & $\overbrace{\frac{\infty}{0}}^{\stackrel{\infty}{0}}$ & 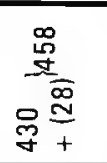 & \\
\hline & & 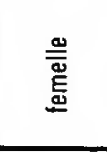 & 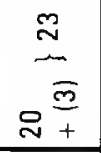 & $\begin{array}{l}= \\
\equiv \\
\equiv\end{array}$ & $\overbrace{m+}^{\bar{m}}$ & $\begin{array}{c}\bar{\sigma} \\
\stackrel{\infty}{\infty} \\
\stackrel{0}{+}\end{array}$ & 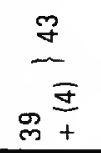 & 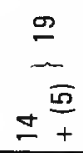 & $\begin{array}{l}\bar{\Xi} \\
\bar{E} \\
\dot{E}=\end{array}$ & \\
\hline & & 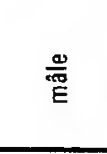 & 电 & $=$ & $\stackrel{\infty}{\infty}$ & $\stackrel{2}{\sim}$ & జ & $\stackrel{20}{\underline{E}}$ & 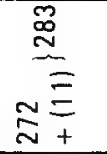 & \\
\hline \multirow{3}{*}{ 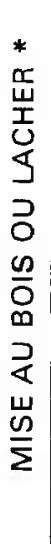 } & & 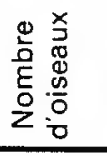 & 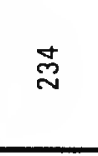 & \pm & $\stackrel{\circ}{\circ}$ & 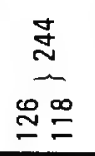 & $\begin{array}{l}\stackrel{\infty}{m} \\
\stackrel{m}{m} \\
\stackrel{\infty}{\infty}\end{array}$ & 窇 & 옹 & \\
\hline & & 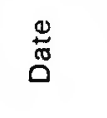 & 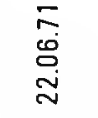 & ㄹ. & $\begin{array}{l}= \\
5 \\
0 \\
0\end{array}$ & $\begin{array}{l}\bar{i} \\
\stackrel{0}{i}\end{array}$ & $\begin{array}{l}\overline{1} \\
8 \\
8 \\
\stackrel{0}{\circ}\end{array}$ & 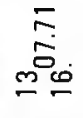 & $\underset{\overparen{E}}{\overrightarrow{5}}$ & \\
\hline & & : & 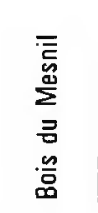 & 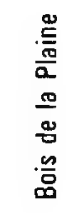 & 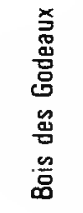 & 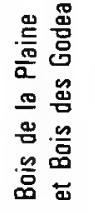 & 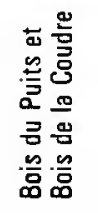 & 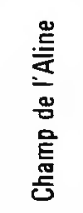 & & \\
\hline & 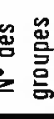 & 总 & - & $N$ & $m$ & $\forall$ & ما & $\omega$ & & \\
\hline
\end{tabular}




\section{RÉSULTATS ET DISCUSSION}

Les principaux résultats pour la saison d'élevage et de chasse 1971-1972 sont rassemblés dans le tableau de la page 350 ; ils doivent être considérés comme partiels car l'expérience se poursuivra pendant la saison suivante.

458 faisans marqués ont été tués ou repris à l'intérieur du territoire central.

Dans le territoire périphérique, on a récolté $5 \mathrm{~B}$ marques qui, étant donné le contrôle partiel, ne représentaient certainement pas la totalitẻ des faisans marqués tués.

\section{Taux de reprise}

Le taux de reprise (rapport du nombre de faisans marqués repris sur le nombre d'oiseaux marqués lâchés) pour le territoire central (1.200 ha) est de $37,9 \%$. Ce taux est significativement plus élevé pour les groupes 1 à 5 que pour le groupe 6 ; cela doit être attribué aux différences des modes d'élevage et de reprise.

Ce résultat est à rapprocher de celui observé en Grande Bretagne pendant la saison 19701971 : The "Game conservancy " a enregistré le taux "relativement élevé " par rapport à la moyenne sur 20 années, de $38,4 \%$, (statistiques portant sur près de 35.000 faisans marqués sur 30 territoires répartis dans les différents comtés).

\section{Sex-ratio}

Le sexage des poussins ou faisandeaux n'a pu être effectué au moment du marquage.

Dans le territoire central, le sex-ratio moyen des faisans marqués tués ou capturés au piège est de 1,65.

Dans l'hypothèse d'un sex-ratio de 1,00 au moment du marquage, le sex-ratio des oiseaux repris ou tués des groupes 1,4 et 5 est significativement supérieur à celui des oiseaux lâchés.

II en est de même si l'on considère l'ensemble des 458 faisans de sexe connu constituant l'ensemble des oiseaux repris ou tués issus de tous les groupes de lâcher.

Par contre le sex-ratio des 809 faisans tués ou repris au piège, autres que les faisans élevés et marqués, est de 0,80 .

Si l'on considère l'ensemble des faisans tués ou repris, marqués ou non marqués le sex-ratio se rapproche de l'unité $(1,03)$.

Comme on a tiré ou capturé sans distinction coqs et poules, cette différence entre le sex-ratio des oiseaux d'élevage marqués et celui des oiseaux non marqués, conduit à plusieurs hypothèses.

1) II peut s'agir d'un déséquilibre existant déjà dans les populations de faisans d'élevage au moment du marquage, renforcé par une mortalité plus grande parmi les poules que parmi les coqs entre la période du marquage et la période de la chasse. coqs.

2) La dispersion des oiseaux d'élevage serait plus grande chez les poules que chez les

C'est l'hypothèse formulée par Blank en Grande Bretagne en 1967. Sur un total de 6.000 faisans marqués, tués à la chasse en 4 ans (1962-1965), sur le territoire de Druids-Lodge (Wiltshire) d'une superficie de 1.500 ha où sont lâchés chaque année environ 4.000 faisandeaux de 7 à 8 semaines sans poule meneuse, Blank obtient un sex-ratio moyen de 1,55.

Dans le cas du territoire d'expérimentation du Gâtinais, le nombre d'oiseaux marqués et de sexe connu tués dans le territoire périphérique est trop petit (39) pour permettre de confirmer cette hypothèse.

On a constaté cependant, que les 12 faisans provenant de l'élevage du Buisson capturés au piège sur les Barres, sont des poules alors que seulement 5 poules contre 11 coqs ont été repris sur les Barres provenant de l'élevage des Barres.

De même 13 poules et seulement 4 coqs provenant de ce dernier élevage ont été tués au Buisson.

Si cette hypothèse était confirmée, le gestionnaire devrait, pour augmenter son taux de reprise, trouver un moyen pour empêcher les poules de se disperser ou lâcher davantage de coqs que de poules. 
La répartition des sexes dans une population de faisan commun comprenant une base de reproduction naturelle et un appoint de faisan d'élevage serait réglée par deux phénomènes agissant en sens contraire et successivement au cours du cycle annuel.

Du lâcher à la fin de l'hiver suivant, les coqs d'élevage resteraient davantage sur la chasse que les poules. Puis, en mars-avril, le comportement territorial se manifestant, les coqs "dominés» se disperseraient.

Pour une meilleure économie du gibier, dans le cas du territoire d'expérimentation, il y aurait lieu de tirer davantage de coqs au moment de la chasse.

Autre conséquence importante, les riverains d'un grand territoire de chasse où l'on fait de l'élevage, pour profiter des coqs dominés se dispersant à partir de mars, devraient lâcher des poules d'élevage.

\section{Dispersion des faisans d'élevage}

Les records de distance atteints par un pọurcentage infime des faisans lâchés n'intéressent pas le gestionnaire de chasse.

En moyenne, pour les lots d'élevage avec poules meneuses, $78 \%$ des faisans marqués et repris en 1971 (157 sur 202) l'ont été dans un cercle d'un km de rayon centré au point de lâcher.

Cette dispersion n'est pas la même dans toutes les directions : ainsi un bois de $0.6 \mathrm{~km}$ de large situé à l'est du territoire central dans un secteur bien contrôlé n'a pratiquement pas été franchi par des faisans lâchés à $0,3 \mathrm{~km}$ de sa lisière (point de lâcher du Bois du Mesnil).

II apparaît une plus grande dispersion en direction des secteurs humides au nord et à l'ouest du territoire d'étude.

\section{Importance de la reproduction naturelle}

Sur le territoire central, le nombre de faisans tués à la chasse ou repris au piège (1.267) est très voisin du nombre d'oiseaux d'élevage lâchés $(1.207) .63,9 \%$ des faisans tués à la chasse ou repris au piège ne proviennent pas des élevages de 1971 (Les Barres, Le Buisson).

Comme on manque de données sur certaines immigrations possibles de faisans d'élevage provenant des territoires situés au sud et à l'est du territoire central d'expérimentation, il faut retenir l'ordre de grandeur : deux faisans sur trois figurant au tableau de chasse sont issus de reproduction naturelle ou bien ont passé en liberté au moins une année dont un hiver.

La poursuite de l'expérience pendant deux ou trois années (durée de renouvellement d'une population de faisans : trois ou quatre ans) et le marquage systématique des lâchers effectués sur les chasses du territoire périphérique permettraient de préciser l'importance de la reproduction naturelle et de comparer les taux de mortalité des oiseaux provenant d'élevage et des oiseaux nés en liberté.

\section{CONCLUSIONS ET APPLICATIONS PRATIQUES}

Une étude complète du devenir des faisans d'élevage, après le lâcher, à condition d'être appliquée à une zone suffisamment étendue et homogène $(2.000$ à 4.000 ha) et d'être suivie pendant trois ou quatre ans au minimum peut fournir de nombreuses informations sur la structure et la dynamique des populations de faisan.

En France, ce genre d'opération coûteuse en personnel spécialisé ne pourrait être menée à bien que dans un petit nombre de territoires reflétant les principales situations écologiques et cynégétiques.

Cependant, le gestionnaire d'un territoire de chasse peut obtenir des informations utiles en marquant ses oiseaux d'élevage et en examinant les tableaux de chasse.

Le marquage est une opération assez rapide puisqu'un groupe de trois opérateurs peut marquer, en 1 heure, 100 à 130 poussins de 8 à 10 jours. 
En recherchant les faisans marqués figurant au tableau de-chasse, le gestionnaire peut calculer le taux de reprise, en suivre la variation d'année en année et en tenir compte dans ses décisions concernant l'élevage, les lâchers, le mode et la pression de chasse, enfin l'aménagement du terrain qui conditionne le maintien des oiseaux d'élevage et le niveau de la reproduction naturelle.

Remarque :

Cotte étude n'a pu étre réalisée que gràce à la compréhension et eu concours des propriátaires at détenteurs du droit de chasse qui nous ont eutorisés à examiner leurs tableaux de chasse et nous ont fait parvenir les marques. Nous les en remercions.

\section{CENTRE TECHNIQUE DU GÉNIE RURAL DES EAUX ET DES FORÊTS \\ Division cynégétique \\ Groupement de Nogent-sur-Vernisson 45290 NOGENT-SUR-VERNISSON}

\section{$\begin{array}{llllllll}\text { I } & \mathbf{N} & \mathbf{F} & \mathbf{O} & \mathbf{R} & \mathbf{M} & \mathbf{A} & \mathbf{T}\end{array}$}

Dans notre mise au point sur le Fomes annosus parue dans la Revue forestière $\left(n^{\circ} 1,1972\right)$, nous avons signalé la bonne résistance du Sapin, notamment le pectiné, au $F$. annosus. Cette résistance semble généralement reconnue et nous avons rapporté, pour illustrer ce fait, les chiffres observés par YDE-ANDERSEN (1968) au Danemark.

Nous pensons cependant que cette notion de résistance du Sapin mérite d'être précisée. En effet, des informations que nous avons recueillies depuis peu nous laissent penser que de nombreux cas de $F$. annosus ont pu être observés en France sur Sapin. II nous a été donné en particulier, grâce à Monsieur R. SCHAEFFER, d'observer personnellement deux cas typiques d'attaque sur Sapin, l'un dans la région de Baume-les-Dames (Doubs) où nous avons isolé le Fomes à la base d'un arbre sur pied par un sondage à la tarière, l'autre dans la région de Besançon (Doubs) où une pourriture typique était visible sur deux souches d'arbres récemment abattus avec une abondante fructification du champignon au niveau des racines.

Les observations actuelles sont malheureusement trop peu nombreuses pour nous permettre de cerner les limites de la résistance du Sapin au $F$. annosus et pour estimer l'importance économique de ce problème. C'est pourquoi il nous serait très utile que les lecteurs de la Revue forestière qui auraient connaissance de la présence du $F$. annosus sur Sapin ou de pourriture de la bille de pied et des racines chez cette essence, puissent nous signaler les cas observés.

\section{DELATOUR}

